\title{
Grappling with Diversity in Livestock-Related, Non-Agriculturist Archaeology in the Light of Genetic Research into the Lactase Persistence Allele, $-14010^{*} \mathrm{C}$, in Southern Africa
}

\author{
Thembi Russell and Faye Lander
}

$\mathbf{1}$

Introduction

The paper by Breton et al. (2014) demonstrating a shared ancestry between an eastern Africa population and the Khoe-speaking Nama of southern Africa has freed archaeologists to consider once again the place of demic diffusion in the spread of the first domestic animals without agriculture, to southern Africa. This follows its unpopularity as an explanation for change amongst the southern Africanist archaeological community in the 199 os and early 2000 (Kinahan, 1991; Sadr, 1998, 2003, 2008; Orton, 2015). It is difficult to separate demic diffusion from cultural diffusion using archaeology, as evidenced in debates worldwide.

This paper looks at the livestock-related, non-agriculturist archaeology in southern Africa in the light of the new genetic insights into the distribution of the lactase persistence allele in southern Africa. We focus on the spread of livestock without agriculture, a process that is connected to Khoe-language speakers. Agro-pastoralism, the spread of farming with speakers of Bantu-languages, is occasionally mentioned for comparative purposes. We briefly review the archaeological evidence for the last 3000 years BP in southern Africa. We then present the modern day southern African distribution of lactose persistence and compare this with the archaeological evidence for livestock-keeping. Finally we consider ethnographic and historic sources for milk-drinking in southern Africa. BP, Southern Africa

Southern Africa is here defined as countries to the south of Congo, the Democratic Republic of Congo and Tanzania. From about 2100 years ago, the 
first, very slight evidence for pottery and domestic stock appears at sites that are conventionally associated with the spread of livestock-keeping without agriculture (referred to as Later Stone Age (LSA) sites in this chapter) (see Lander and Russell (2018) for a detailed review of the data from $55^{1}$ вC to AD 1056). The most securely identified and directly dated sheep specimen derives from the site Spoegrivier on the western half of southern Africa and dates to around 2100 years ago (Coutu et al., 2021). The earliest appearance of domestic cattle at sites conventionally associated with the spread of farmers speaking Bantu-languages occurs from about 1750 вР onwards (referred to as farmer sites in this chapter). At around 1500 years BP livestock counts reach a peak at Later Stone Age sites, with evidence of caprines outweighing cattle, whilst in the summer rainfall area, on the eastern side of southern Africa, many farmer sites have evidence of livestock-keeping in the form of cattle bones (Figure 6.1). From 1300 years BP, the number and distribution of farmer-related sites steadily increase, whilst the number of Later Stone Age livestock-related sites remain constant from this period onwards. Notably LSA sites have consistently low numbers of domestic livestock (Russell and Lander, 2015). Cattle are rare. The total cattle count (MNI) at all LSA sites is just 21, whilst that for caprines (mainly sheep) is 365 (Russell and Lander, 2015).

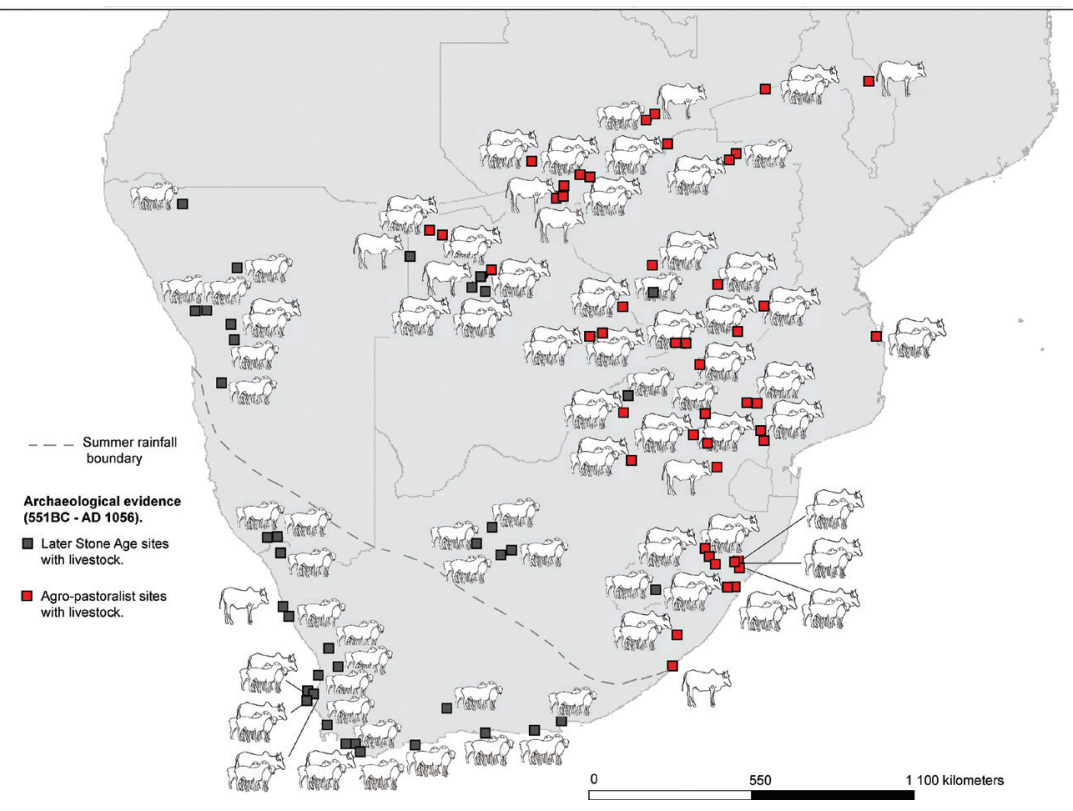

FIGURE 6.1 Archaeological evidence for livestock in Southern Africa, 551 BC to AD 1058 
Contemporary with the first appearance of livestock is pottery. However, attempts to link it with the spread of livestock at Later Stone Age sites based on stylistic analysis have been unfruitful (Sadr and Sampson, 2006; Sadr, 2008; see also Smith, 2008, 2017). Lander and Russell (2020) suggest that pottery spread rapidly amongst hunter-gatherers from its first appearance in a process of cultural diffusion, which might also have carried domestic livestock, particularly sheep, across South Africa along already established exchange networks (see also Sadr, 2004; Russell, 2017).

Distribution of the Southern African Lactase Persistence Allele, $-14010^{*} \mathrm{C}$ Compared to the Archaeological Evidence for Livestock

The presence of the east African lactase persistence allele, $-14010^{*} \mathrm{C}$, amongst present-day southern African populations is important because it signifies the presence of a proto-historic, fresh-milk-drinking pastoral population in southern Africa, and in the case of the Nama, a degree of relatedness to an East African source population (Breton et al., 2014; Macholdt et al., 2014). In southern Africa, 59 ethnic population groups have been screened for the LP allele (Table 6.1 and Figure 6.2) (Coelho et al., 20o9; Tornianen et al., 20o9; Breton et al., 2014; Macholdt et al., 2014; Jones et al., 2015; Pinto et al., 2016). These include Afrikaans-, Khoesan- and Bantu- language-speakers from agro-pastoralist, hunter-gatherer and agriculturist communities. The geographical coverage includes parts of Angola, Namibia, Botswana, Zambia, Kingdom of Eswatini, Mozambique and South Africa (Figure 6.3).

The lactase persistence allele reflects the continuous consumption of fresh milk from one generation to the next (Tishkoff et al., 2007; Breton et al., 2014; Ranciaro et al., 2014). Its presence is thus indicative of a group that either keeps livestock and drinks fresh milk or gets fresh milk regularly from a continuous relationship with livestock-keepers.

In the review of LP $-14010^{*} \mathrm{C}$ allele amongst extant southern African population groups, twelve have the allele at frequencies of $10 \%$ or above (Table 6.1). The highest incidence is found amongst the Namibian Nama Khoe-speaking pastoralists (35.7\%) (Breton et al., 2014; Macholdt et al., 2014). Nine of these groups are found in northern/north-western parts of southern Africa. The remaining three are distinctive. Two are eastern Bantu-language speaking groups (Map Code 15 and 35, Figure 6.4). The third is an Afrikaans-speaking community of mixed Khoesan ancestry, sampled in the western Cape, South Africa (Breton et al., 2014) (map code 3, Figure 6.4). 
TABLE 6.1 The frequency of the East African - lactase persistence allele in Southern Africa

\begin{tabular}{|c|c|c|c|}
\hline $\begin{array}{l}\text { Map } \\
\text { code }\end{array}$ & Ethnic group & $\begin{array}{l}\text { Subsistence base, language } \\
\text { group, ref. }\end{array}$ & $\mathrm{N}$ of individuals \\
\hline 1 & Nama & $\begin{array}{l}\text { Pastoralist } \\
\text { Khoe } \\
\text { Breton et al. } 2014\end{array}$ & 22 \\
\hline 2 & Askham Coloured & $\begin{array}{l}\text { Not provided } \\
\text { Khoe - North } \\
\text { Breton et al. } 2014\end{array}$ & 20 \\
\hline 3 & Wellington Coloured & $\begin{array}{l}\text { Not provided } \\
\text { Afrikaans } \\
\text { Breton et al. } 2014\end{array}$ & 20 \\
\hline 4 & Colesberg Coloured & $\begin{array}{l}\text { Not provided } \\
\text { Afrikaans } \\
\text { Breton et al. } 2014\end{array}$ & 20 \\
\hline 5 & /Gui and //Gana & $\begin{array}{l}\text { Hunter-gatherer } \\
\text { Khoe } \\
\text { Breton et al. } 2014\end{array}$ & 20 \\
\hline 5 & //Gana & $\begin{array}{l}\text { Hunter-gatherer } \\
\text { Khoe } \\
\text { Macholdt et al. } 2014\end{array}$ & 10 \\
\hline 5 & /Gui & $\begin{array}{l}\text { Hunter-gatherer } \\
\text { Khoe } \\
\text { Macholdt et al. } 2014\end{array}$ & 17 \\
\hline 6 & Khwe & $\begin{array}{l}\text { Hunter-gatherer } \\
\text { Khoe } \\
\text { Breton et al. } 2014\end{array}$ & 19 \\
\hline 6 & Khwe & $\begin{array}{l}\text { Hunter-gatherer } \\
\text { Khoe } \\
\text { Breton et al. } 2014\end{array}$ & 19 \\
\hline 7 & Ju/'hoansi & $\begin{array}{l}\text { Hunter-gatherer } \\
\text { Ju - Northeast - Ju/'hoan } \\
\text { Breton et al. } 2014\end{array}$ & 20 \\
\hline 8 & $\begin{array}{l}\text { Ju|hoan } \\
\text { North }\end{array}$ & $\begin{array}{l}\text { Hunter-gatherer } \\
\text { Kx'a } \\
\text { Macholdt et al. } 2014\end{array}$ & 21 \\
\hline
\end{tabular}




$\begin{array}{lllll}\text { Sample region } & \begin{array}{l}\text { Decimal } \\ \text { latitude }\end{array} & \begin{array}{l}\text { Decimal } \\ \text { longitude }\end{array} & \begin{array}{l}-14010^{*} \mathrm{C} \\ \text { freq. }\end{array} & \text { Ref. }\end{array}$

Windhoek, Namibia $\quad-22,5624 \quad 17,06599 \quad 0,357 \quad$ Breton et al. (2014) Macholdt et al. (2014)

Askham, Northern Cape, $\quad-26,9834 \quad 20,78333 \quad$ 0,225 Breton et al. (2014)

South Africa

$\begin{array}{lllll}\begin{array}{l}\text { Wellington, Western } \\ \text { Cape, South Africa }\end{array} & -33,6818 & 19,01023 & 0,1 & \text { Breton } \text { et al. (2014) } \\ \begin{array}{l}\text { Colesberg, Northern } \\ \text { Cape, South Africa }\end{array} & -30,8388 & 25,07629 & 0,025 & \text { Breton } \text { et al.(2014) }\end{array}$

Cape, South Africa

Kutse Game Reserve, $\quad-23,4223 \quad 24,04879 \quad$ 0,071 $\quad$ Breton et al. (2014)

Kalahari, Botswana

Kutse Game Reserve, $\quad-23,4223 \quad 24,04879 \quad$ 0,2 $\quad$ Macholdt et al. (2014)

Kalahari, Botswana

$\begin{array}{lcccc}\begin{array}{l}\text { Kutse Game Reserve, } \\ \text { Kalahari, Botswana }\end{array} & -23,4223 & 24,04879 & 0,088 & \text { Macholdt } \text { et al. (2014) } \\ \begin{array}{l}\text { Schmidtsdrif Northern } \\ \text { Cape, South Africa }\end{array} & -28,8134 & 24,10132 & 0,029 & \text { Breton } \text { et al. (2014) }\end{array}$

Rootfontein, Namibia $\quad-19,6113 \quad 18,10939 \quad$ O,029 Breton et al. (2014)

Tsumkwe, Namibia $\quad-19,6009 \quad 20,50384 \quad 0,029 \quad$ Breton et al. (2014)

North West, Botswana $\quad-20,5814 \quad 21,67013 \quad$ o, $\quad 24 \quad$ Macholdt et al. (2014) 
TABLE 6.1 The Frequency of the East African - lactase persistence allele in Southern Africa (cont.)

\begin{tabular}{|c|c|c|c|}
\hline $\begin{array}{l}\text { Map } \\
\text { code }\end{array}$ & Ethnic group & $\begin{array}{l}\text { Subsistence base, language } \\
\text { group, ref. }\end{array}$ & $\mathrm{N}$ of individuals \\
\hline 9 & $\begin{array}{l}\text { Ju|hoan } \\
\text { South }\end{array}$ & $\begin{array}{l}\text { Hunter-gatherer } \\
\text { Kx'a } \\
\text { Macholdt et al. } 2014\end{array}$ & 26 \\
\hline 10 & !Xun & $\begin{array}{l}\text { Hunter-gatherer } \\
\text { Ju - Northwest - /Xũu } \\
\text { Breton et al. } 2014\end{array}$ & 20 \\
\hline 10 & !Xun & $\begin{array}{l}\text { Hunter-gatherer } \\
\text { Ju - Northwest - /Xũu } \\
\text { Breton et al. } 2014\end{array}$ & - \\
\hline 20 & !Xuun & $\begin{array}{l}\text { Hunter-gatherer } \\
\text { Kx'a } \\
\text { Macholdt et al. } 2014\end{array}$ & 19 \\
\hline 47 & !Xuun & $\begin{array}{l}\text { Hunter-gatherer } \\
\text { Kx'a } \\
\text { Pinto et al. } 2016\end{array}$ & - \\
\hline 11 & Karretjie People & $\begin{array}{l}\text { Hunter-gatherer and } \\
\text { herder } \\
\text { /Xam descendants } \\
\text { Breton et al. } 2014\end{array}$ & 20 \\
\hline 12 & $\neq$ Khomani & $\begin{array}{l}\text { Hunter-gatherer herders } \\
\text { Germanic Tuu } \\
\text { Breton et al. } 2014\end{array}$ & 20 \\
\hline 13 & Herero & $\begin{array}{l}\text { Farmer and herder } \\
\text { western Bantu } \\
\text { Breton et al. } 2014\end{array}$ & 14 \\
\hline 14 & Herero & $\begin{array}{l}\text { Pastoralist } \\
\text { western Bantu } \\
\text { Macholdt et al. } 2014\end{array}$ & 21 \\
\hline 38 & $\begin{array}{l}\text { Kuvale/ } \\
\text { Herero }\end{array}$ & $\begin{array}{l}\text { Pastoralist } \\
\text { west Savanna Bantu } \\
\text { Coelho } \text { et al. } 2009\end{array}$ & \\
\hline 42 & Kuvale & $\begin{array}{l}\text { Farming with some } \\
\text { pastoralism } \\
\text { west Savanna Bantu } \\
\text { Pinto et al. } 2016\end{array}$ & - \\
\hline
\end{tabular}




\begin{tabular}{|c|c|c|c|c|}
\hline Sample region & $\begin{array}{l}\text { Decimal } \\
\text { latitude }\end{array}$ & $\begin{array}{l}\text { Decimal } \\
\text { longitude }\end{array}$ & $\begin{array}{l}-14010^{*} \mathrm{C} \\
\text { freq. }\end{array}$ & Ref. \\
\hline $\begin{array}{l}\text { Ghanzi, Botswana border } \\
\text { with Namibia }\end{array}$ & $-21,1943$ & 21,06119 & o,o58 & Macholdt et al. (2014) \\
\hline $\begin{array}{l}\text { Schmidtsdrif, Northern } \\
\text { Cape, South Africa }\end{array}$ & $-28,8134$ & 24,10119 & o,o38 & Breton et al. (2014) \\
\hline Grootfontein, Namibia & $-19,6113$ & 18,10939 & o,o38 & Breton et al. (2014) \\
\hline Nyae Nyae, Namibia & $-19,7057$ & 20,49703 & o,o53 & Macholdt et al. (2014) \\
\hline
\end{tabular}

\begin{tabular}{|c|c|c|c|c|}
\hline Mupa, Angola & $-16,1831$ & 15,76708 & 0,014 & Pinto et al. (2016) \\
\hline Colesberg, Northern & $-30,8388$ & 25,07629 & 0,083 & Breton et al. (2014) \\
\hline
\end{tabular}

Cape, South Africa

$\begin{array}{llll}\text { Askham, Northern Cape, } & -26,9834 & 20,78333 & 0,11 \quad \text { Breton } \text { et al. (2014) } \\ \text { South Africa } & \end{array}$

Windhoek, Namibia $\quad-17,0624 \quad 17,06599 \quad$ o $\quad$ Breton et al. (2014)

$\begin{array}{lllll}\text { Windhoek, Namibia } & -22,5624 & 17,06599 & 0,071 & \begin{array}{l}\text { Macholdt } \text { et al. (2014) (see } \\ \text { Torniainen } \text { et al. 2009 and }\end{array} \\ & & & \text { Breton } \text { et al. 2014) }\end{array}$

\begin{tabular}{|c|c|c|c|c|}
\hline $\begin{array}{l}\text { Namibe, Angola } \\
\text { [1 sample location] }\end{array}$ & $-14,5436$ & 13,10684 & o,037 & $\begin{array}{l}\text { Pinto et al. (2016) (see } \\
\text { Coelho et al. 2009) }\end{array}$ \\
\hline
\end{tabular}


TABLE 6.1 The Frequency of the East African - lactase persistence allele in Southern Africa (cont.)

\begin{tabular}{|c|c|c|c|}
\hline $\begin{array}{l}\text { Map } \\
\text { code }\end{array}$ & Ethnic group & $\begin{array}{l}\text { Subsistence base, language } \\
\text { group, ref. }\end{array}$ & $\mathrm{N}$ of individuals \\
\hline 42 & Kuvale & $\begin{array}{l}\text { Farming with some } \\
\text { pastoralism } \\
\text { west Savanna Bantu } \\
\text { Pinto et al. } 2016\end{array}$ & - \\
\hline 42 & Kuvale & $\begin{array}{l}\text { Farming with some } \\
\text { pastoralism } \\
\text { west Savanna Bantu } \\
\text { Pinto et al. } 2016\end{array}$ & - \\
\hline 15 & Sotho-Tswana and Zulu & $\begin{array}{l}\text { Agropastoralist farmer } \\
\text { southeastern Bantu } \\
\text { Breton et al. } 2014\end{array}$ & $\begin{array}{l}16 \text { (Sotho-Tswana) } 25 \\
\text { (Zulu) }\end{array}$ \\
\hline 16 & Taa-East & $\begin{array}{l}\text { Hunter-gatherer } \\
\text { Tuu } \\
\text { Macholdt et al. } 2014\end{array}$ & 11 \\
\hline 17 & Taa-North & $\begin{array}{l}\text { Hunter-gatherer } \\
\text { Tuu } \\
\text { Macholdt et al. } 2014\end{array}$ & 11 \\
\hline 18 & Taa-West & $\begin{array}{l}\text { Hunter-gatherer } \\
\text { Tuu } \\
\text { Macholdt et al. } 2014\end{array}$ & 20 \\
\hline 19 & $\ddagger$ Hoan & $\begin{array}{l}\text { Hunter-gatherer } \\
\text { Kx'a } \\
\text { Macholdt et al. } 2014\end{array}$ & 7 \\
\hline 21 & $\|$ Ani & $\begin{array}{l}\text { Hunter-gatherer } \\
\text { Khoe } \\
\text { Macholdt et al. } 2014\end{array}$ & 11 \\
\hline 22 & Buga & $\begin{array}{l}\text { Hunter-gatherer } \\
\text { Khoe } \\
\text { Macholdt et al. } 2014\end{array}$ & 9 \\
\hline 23 & $\|$ Хo & $\begin{array}{l}\text { Hunter-gatherer } \\
\text { Khoe } \\
\text { Macholdt et al. } 2014\end{array}$ & 19 \\
\hline
\end{tabular}




$\begin{array}{lllll}\text { Sample region } & \text { Decimal } & \text { Decimal } & -14010^{*} \mathrm{C} & \text { Ref. } \\ & \text { latitude } & \text { longitude } & \text { freq. } & \end{array}$

\begin{tabular}{|c|c|c|c|c|}
\hline $\begin{array}{l}\text { Namibe, Angola [2 } \\
\text { sample location] }\end{array}$ & $-15,3969$ & 12,83899 & o,o37 & $\begin{array}{l}\text { Pinto et al.(2016) (see } \\
\text { Coelho et al. 2009) }\end{array}$ \\
\hline
\end{tabular}

\begin{tabular}{|c|c|c|c|c|}
\hline $\begin{array}{l}\text { Namibe, Angola [3 } \\
\text { sample location] }\end{array}$ & $-15,5753$ & 12,76128 & o,o37 & $\begin{array}{l}\text { Pinto et al. (2016) (see } \\
\text { Coelho et al. 2009) }\end{array}$ \\
\hline
\end{tabular}

$\begin{array}{lllll}\text { Various regions, South } & -27,9865 & 29,86269 & 0,1 & \text { Breton } \text { et al. (2014) } \\ \text { Africa } & & & \\ & & & \\ \text { Kgalagadi, Botswana } & -24,3548 & 22,81767 & \text { 0,045 } & \text { Macholdt et al. (2014) }\end{array}$

Kgalagadi, Botswana $\quad-23,59 \quad 21,61049 \quad$ Macholdt et al. (2014)

Kgalagadi, Botswana $\quad-24,7803 \quad 20,10442 \quad 0,025 \quad$ Macholdt et al. (2014)

Kgalagadi, Botswana $\quad-24,3368 \quad 22,34 \quad$ Macholdt et al. (2014)

North West, Botswana $\quad-18,5167 \quad 21,94934 \quad$ o,o91 $\quad$ Macholdt et al. (2014)

North West, Botswana $\quad-18,3716 \quad 21,85806 \quad$ o $\quad$ Macholdt et al. (2014)

$\begin{array}{llll}\text { Caprivi Strip } \quad-17,9231 & 22,72381 & \text { o,o79 } & \text { Macholdt } \text { et al. }(2014)\end{array}$


TABLE 6.1 The Frequency of the East African - lactase persistence allele in Southern Africa (cont.)

\begin{tabular}{|c|c|c|c|}
\hline $\begin{array}{l}\text { Map } \\
\text { code }\end{array}$ & Ethnic group & $\begin{array}{l}\text { Subsistence base, language } \\
\text { group, ref. }\end{array}$ & $\mathrm{N}$ of individuals \\
\hline 24 & Damara & $\begin{array}{l}\text { Hunter-gatherer and } \\
\text { pastoralist } \\
\text { Khoe } \\
\text { Macholdt et al. } 2014\end{array}$ & 34 \\
\hline 25 & Haillom & $\begin{array}{l}\text { Hunter-gatherer } \\
\text { Khoe } \\
\text { Macholdt et al. } 2014\end{array}$ & 40 \\
\hline 26 & Naro & $\begin{array}{l}\text { Hunter-gatherer } \\
\text { Khoe } \\
\text { Macholdt et al. } 2014\end{array}$ & 19 \\
\hline 27 & Shua & $\begin{array}{l}\text { Hunter-gatherer } \\
\text { Khoe } \\
\text { Macholdt et al. } 2014\end{array}$ & 27 \\
\hline 28 & Tshwa & $\begin{array}{l}\text { Hunter-gatherer } \\
\text { Khoe } \\
\text { Macholdt et al. } 2014\end{array}$ & 15 \\
\hline 29 & Himba & $\begin{array}{l}\text { Pastoralist } \\
\text { west Savanna Bantu } \\
\text { Macholdt et al. } 2014\end{array}$ & 16 \\
\hline 41 & Himba & $\begin{array}{l}\text { Pastoralist } \\
\text { west Savanna Bantu } \\
\text { Pinto et al. 2016; Macholdt } \\
\text { et al. } 2014\end{array}$ & - \\
\hline 41 & Himba & $\begin{array}{l}\text { Pastoralist } \\
\text { west Savanna Bantu } \\
\text { Pinto et al. 2016; Macholdt } \\
\text { et al. } 2014\end{array}$ & - \\
\hline 30 & Kgalagadi & $\begin{array}{l}\text { Agropastoralist } \\
\text { southeastern Bantu } \\
\text { Macholdt et al. } 2014\end{array}$ & 20 \\
\hline 31 & Tonga & $\begin{array}{l}\text { Agriculturist } \\
\text { southeastern Bantu } \\
\text { Macholdt et al. } 2014\end{array}$ & 17 \\
\hline
\end{tabular}




$\begin{array}{lllll}\text { Sample region } & \text { Decimal } & \text { Decimal } & -14010^{*} \mathrm{C} & \text { Ref. } \\ & \text { latitude } & \text { longitude } & \text { freq. } & \end{array}$

Kunene Region, Namibia $\quad-20,4608 \quad 14,01402 \quad 0,044 \quad$ Macholdt et al. (2014)

Kunene Region, Namibia $\quad-19,6687 \quad 14,70835 \quad$ 0,088 $\quad$ Macholdt et al. (2014)

$\begin{array}{lllll}\text { Ghanzi, Botswana } & -22,01 & 21,23226 & 0,053 & \text { Macholdt et al. (2014) } \\ \text { Makgadikgadi, Botswana } & -20,5509 & 25,8103^{1} & \text { 0, } 074 & \text { Macholdt } \text { et al. }(2014)\end{array}$

$\begin{array}{lllll}\text { Makgadikgadi, Botswana } & -21,3433 & 26,04308 & 0,167 & \text { Macholdt } \text { et al. (2014) } \\ \text { Skeleton Coast, Namibia } & -19,5689 & 13,67961 & 0,125 & \text { Macholdt } \text { et al. }(2014)\end{array}$

\begin{tabular}{|c|c|c|c|c|}
\hline $\begin{array}{l}\text { Namibe, Angola [1 } \\
\text { sample location] }\end{array}$ & $-17,0017$ & 12,43598 & ०,०87 & $\begin{array}{l}\text { Pinto et al. (2016) (see } \\
\text { Macholdt et al. 2014) }\end{array}$ \\
\hline
\end{tabular}

\begin{tabular}{|c|c|c|c|c|}
\hline $\begin{array}{l}\text { Namibe, Angola [2 } \\
\text { sample location] }\end{array}$ & $-16,8911$ & $12,4359^{8}$ & o,o87 & $\begin{array}{l}\text { Pinto et al. (2016) (see } \\
\text { Macholdt et al. 2014) }\end{array}$ \\
\hline
\end{tabular}

$\begin{array}{lllll}\text { Kgalagadi, Botswana } & -24,7289 & 22,70295 & \circ & \text { Macholdt } \text { et al. }(2014) \\ \text { southern Zambia } & -17,7183 & 26,82519 & \text { O } & \text { Macholdt } \text { et al. }(2014)\end{array}$


TABLE 6.1 The Frequency of the East African - lactase persistence allele in Southern Africa (cont.)

\begin{tabular}{|c|c|c|c|}
\hline $\begin{array}{l}\text { Map } \\
\text { code }\end{array}$ & Ethnic group & $\begin{array}{l}\text { Subsistence base, language } \\
\text { group, ref. }\end{array}$ & $\mathrm{N}$ of individuals \\
\hline $3^{2}$ & Tswana & $\begin{array}{l}\text { Agropastoralist } \\
\text { southeastern Bantu } \\
\text { Macholdt et al. } 2014\end{array}$ & 18 \\
\hline 33 & Nkoya & $\begin{array}{l}\text { Agropastoralist } \\
\text { eastern Bantu } \\
\text { Macholdt et al. } 2014\end{array}$ & 16 \\
\hline 34 & Wambo & $\begin{array}{l}\text { Agriculturist } \\
\text { western Bantu } \\
\text { Macholdt et al. } 2014\end{array}$ & 8 \\
\hline 35 & !Xhosa & $\begin{array}{l}\text { Agropastoralist } \\
\text { southeastern Bantu } \\
\text { Torniainen et al. } 2009\end{array}$ & 109 \\
\hline 35 & !Xhosa & $\begin{array}{l}\text { Agropastoralist } \\
\text { southeastern Bantu } \\
\text { Ranciaro et al. } 2014\end{array}$ & 16 \\
\hline 36 & Venda & $\begin{array}{l}\text { Agropastoralist } \\
\text { southeastern Bantu } \\
\text { Ranciaro et al. } 2014\end{array}$ & 18 \\
\hline 37 & Ovim-bundu & $\begin{array}{l}\text { Mostly agriculturists } \\
\text { (cattle raising not crucial } \\
\text { for subsistence) } \\
\text { west Savanna Bantu } \\
\text { Coelho et al. } 2009\end{array}$ & - \\
\hline 39 & Nyaneka-Nkhumbi & $\begin{array}{l}\text { Agropastoralist } \\
\text { (predominantly cattle } \\
\text { raisers) } \\
\text { west Savanna Bantu } \\
\text { Coelho et al. } 2009\end{array}$ & - \\
\hline 40 & Guang-uela & $\begin{array}{l}\text { Agropastoralist } \\
\text { west Savanna Bantu } \\
\text { Coelho et al. } 2009\end{array}$ & - \\
\hline
\end{tabular}




\section{Sample region}

Kweneng, southern

Botswana

Zambia

$-14,6633$

25,50005

o,o31

Macholdt et al. (2014)

\section{latitude longitude freq.}

Macholdt et al. (2014) (see

Breton et al. 2014)

Northern Nambia

$-17,7569 \quad 16,63925$

Macholdt et al. (2014)

Eastern Cape and western Cape, South

$-33,1477 \quad 26,54454 \quad 0,128$

Torniainen et al. (2009)

Africa

Western Cape, South

$-34,079$

19,10157

0,1429

Ranciaro et al. (2014)

Africa

Thohoyandou, South

Africa

$-23,1016 \quad 30,59716 \quad 0$

Ranciaro et al. (2014)

Namibe, Angola

$-14,733$

$13,245^{21}$

0,01

Coelho et al. (2009)

Namibe, Angola

$-15,1194$

12,71634

$\mathrm{O}, \mathrm{O} 3$

Coelho et al. (2009)

Namibe, Angola

$-15,44$

12,92882

o

Coelho et al. (2009) 
TABLE 6.1 The Frequency of the East African - lactase persistence allele in Southern Africa (cont.)

\begin{tabular}{|c|c|c|c|}
\hline $\begin{array}{l}\text { Map } \\
\text { code }\end{array}$ & Ethnic group & $\begin{array}{l}\text { Subsistence base, language } \\
\text { group, ref. }\end{array}$ & $\mathrm{N}$ of individuals \\
\hline 43 & Kwepe & $\begin{array}{l}\text { Shepherd/livestock-keeper } \\
\text { Khoe-Kwadi (recently } \\
\text { replaced by Kuvale) } \\
\text { Pinto et al. } 2016\end{array}$ & - \\
\hline 43 & Kwepe & $\begin{array}{l}\text { Shepherd/livestock-keeper } \\
\text { Khoe-Kwadi (recently } \\
\text { replaced by Kuvale) } \\
\text { Pinto et al. } 2016\end{array}$ & - \\
\hline 44 & Kwisi & $\begin{array}{l}\text { Hunter-gatherer (recently } \\
\text { cattle-keepers) } \\
\text { west Savanna Bantu } \\
\text { Pinto et al. } 2016\end{array}$ & - \\
\hline 44 & Kwisi & $\begin{array}{l}\text { Hunter-gatherer (recently } \\
\text { cattle-keepers) } \\
\text { west Savanna Bantu } \\
\text { Pinto et al. } 2016\end{array}$ & - \\
\hline 45 & Twa & $\begin{array}{l}\text { Hunter-gatherer (recently } \\
\text { cattle-keepers) } \\
\text { west Savanna Bantu } \\
\text { Pinto et al. } 2016\end{array}$ & - \\
\hline 45 & Twa & $\begin{array}{l}\text { Hunter-gatherer (recently } \\
\text { cattle-keepers) } \\
\text { west Savanna Bantu } \\
\text { Pinto et al. } 2016\end{array}$ & - \\
\hline 46 & Tjimba & $\begin{array}{l}\text { Hunter-gatherer (cattle- } \\
\text { less pastoralists) } \\
\text { west Savanna Bantu } \\
\text { Pinto et al. } 2016\end{array}$ & - \\
\hline 48 & Yao & $\begin{array}{l}\text { Agriculturist } \\
\text { Kaskazi-speaking } \\
\text { Pinto et al. } 2016\end{array}$ & - \\
\hline 49 & Nyanja & $\begin{array}{l}\text { Agriculturist } \\
\text { southeastern Bantu } \\
\text { Pinto et al. } 2016\end{array}$ & - \\
\hline
\end{tabular}


Sample region

Namibe, Angola [1

sample location]

Namibe, Angola [2

sample location]

Namibe, Angola [1

sample location]

Namibe, Angola [2

sample location]

Namibe, Angola [1

sample location]

Namibe, Angola [2

sample location]

$\begin{array}{llll}\text { Decimal } & \text { Decimal } & -14010^{*} \mathrm{C} & \text { Ref. } \\ \text { latitude } & \text { longitude } & \text { freq. } & \end{array}$

$-15,8062 \quad 12,1081 \quad 0,044 \quad$ Pinto et al. $(2016)$
$-15,8274 \quad 12,45^{268} \quad$ o,044 Pinto et al. $(2016)$

$-15,7196 \quad 12,45046 \quad 0,175 \quad$ Pinto et al. $(2016)$

$-15,5978 \quad 12,73733 \quad 0,175 \quad$ Pinto et al. $(2016)$


TABLE 6.1 The Frequency of the East African - lactase persistence allele in Southern Africa (cont.)

\begin{tabular}{|c|c|c|c|}
\hline $\begin{array}{l}\text { Map } \\
\text { code }\end{array}$ & Ethnic group & $\begin{array}{l}\text { Subsistence base, language } \\
\text { group, ref. }\end{array}$ & $\mathrm{N}$ of individuals \\
\hline $5^{\circ}$ & Makua & $\begin{array}{l}\text { Agriculturist } \\
\text { southeastern Bantu } \\
\text { Pinto et al. } 2016\end{array}$ & - \\
\hline $5^{1}$ & Tswa & $\begin{array}{l}\text { Mixed agriculturist } \\
\text { southeastern Bantu } \\
\text { Pinto et al. } 2016\end{array}$ & - \\
\hline $5^{2}$ & Shangaan & $\begin{array}{l}\text { Mixed agriculturist } \\
\text { southeastern Bantu } \\
\text { Pinto et al. } 2016\end{array}$ & - \\
\hline 53 & Chopi & $\begin{array}{l}\text { Agriculturist } \\
\text { southeastern Bantu } \\
\text { Coelho et al. } 2009\end{array}$ & 3 \\
\hline 54 & Ronga & $\begin{array}{l}\text { Agriculturist } \\
\text { southeastern Bantu } \\
\text { Coelho et al. } 2009\end{array}$ & 15 \\
\hline 55 & Sena & $\begin{array}{l}\text { Agriculturist } \\
\text { southeastern Bantu } \\
\text { Coelho } \text { et al. } 2009\end{array}$ & 2 \\
\hline $5^{6}$ & Ndau & $\begin{array}{l}\text { Mixed agriculturist } \\
\text { southeastern Bantu } \\
\text { Coelho } \text { et al. } 2009\end{array}$ & 15 \\
\hline 57 & Chwabo & $\begin{array}{l}\text { Agriculturist } \\
\text { southeastern Bantu } \\
\text { Coelho } \text { et al. } 2009\end{array}$ & 4 \\
\hline $5^{8}$ & Shona & $\begin{array}{l}\text { Mixed agriculturist } \\
\text { southeastern Bantu } \\
\text { Coelho et al. } 2009\end{array}$ & 1 \\
\hline 59 & Swazi & $\begin{array}{l}\text { Agropastoralist } \\
\text { southeastern Bantu } \\
\text { Segal } \text { et al. } 1987\end{array}$ & 12 \\
\hline
\end{tabular}




\begin{tabular}{|c|c|c|c|c|}
\hline Sample region & $\begin{array}{l}\text { Decimal } \\
\text { latitude }\end{array}$ & $\begin{array}{l}\text { Decimal } \\
\text { longitude }\end{array}$ & $\begin{array}{l}-14010^{*} \mathrm{C} \\
\text { freq. }\end{array}$ & Ref. \\
\hline Mozambique & $-15,2267$ & 39,23246 & o & $\begin{array}{l}\text { Pinto et al. (2016) (see Alves } \\
\text { et al. 2011) }\end{array}$ \\
\hline Mozambique & $-21,4492$ & 35,00139 & o & $\begin{array}{l}\text { Pinto et al. (2016) (see Alves } \\
\text { et al. 2011) }\end{array}$ \\
\hline Mozambique & $-24,895^{8}$ & 32,98332 & 0,022 & $\begin{array}{l}\text { Pinto et al. (2016) (see Alves } \\
\text { et al. 2011) }\end{array}$ \\
\hline Mozambique & $-24,792$ & 34,37146 & o & $\begin{array}{l}\text { Coehlo et al. (2009) (see } \\
\text { Alves et al. 2011; Pinto et al. } \\
2016)\end{array}$ \\
\hline Southern Mozambique & $-26,345$ & 32,50994 & o & $\begin{array}{l}\text { Coehlo et al. (2009) (see } \\
\text { Alves et al. 2011; Pinto et al. } \\
2016)\end{array}$ \\
\hline Mozambique & $-17,7261$ & 34,95491 & o & $\begin{array}{l}\text { Coehlo et al. (2009) (see } \\
\text { Alves et al. 2011; Pinto et al. } \\
2016)\end{array}$ \\
\hline Mozambique & $-19,3029$ & 34,55766 & o & $\begin{array}{l}\text { Coehlo et al. (2009) (see } \\
\text { Alves et al. 2011; Pinto et al. } \\
2016)\end{array}$ \\
\hline Mozambique & $-17,1928$ & 36,45974 & o & $\begin{array}{l}\text { Coehlo et al. (2009) (see } \\
\text { Alves et al.2011; Pinto et al. } \\
2016)\end{array}$ \\
\hline Harare, Zimbabwe & $-17,9233$ & 30,95064 & o & Coelho et al. (2009) \\
\hline Mbabane, Eswatini & $-26,3323$ & $31,15^{249}$ & O & $\begin{array}{l}\text { Segal et al. (1987) (see } \\
\text { Holden and Mace 20o9). }\end{array}$ \\
\hline
\end{tabular}




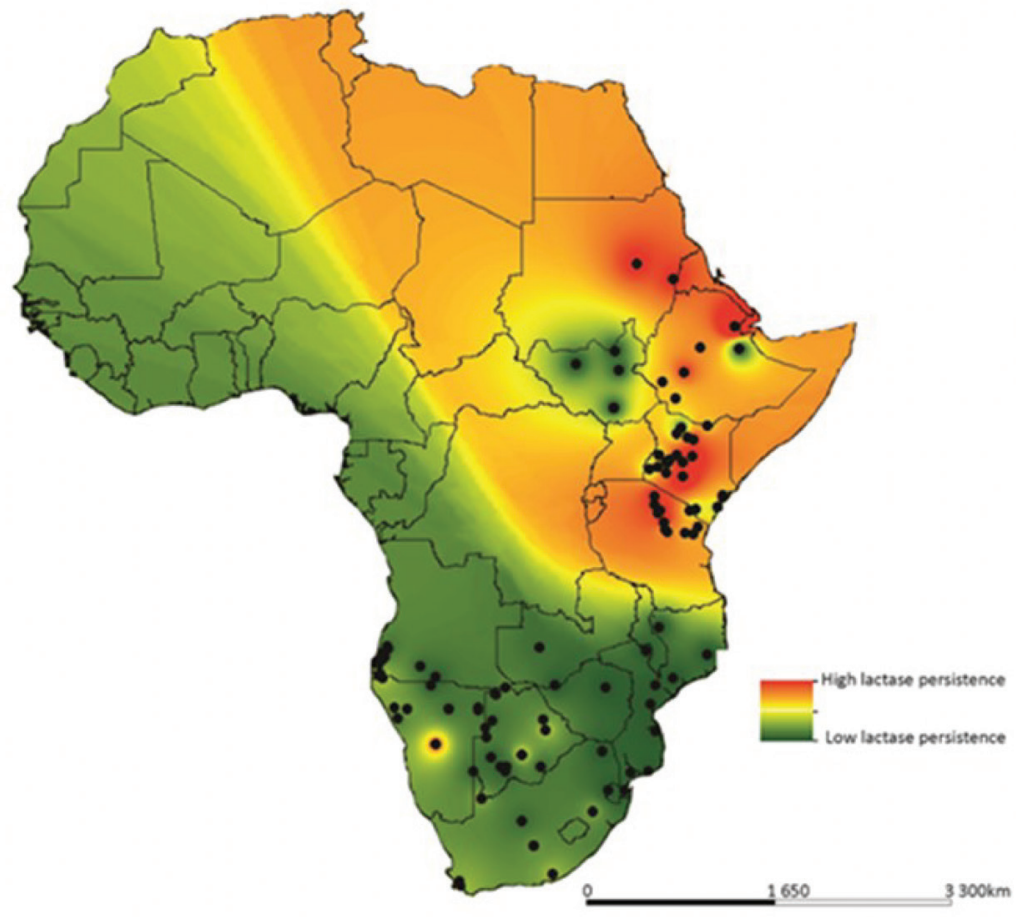

FIGURE 6.2 Map showing the distribution of modern East and Southern African populations screened for the East African LP allele $-14010^{*} \mathrm{C}$

Three patterns in the distribution of the LP allele stand out. These are considered in relation to the archaeological evidence for livestock-keeping.

1. The lactase persistence allele occurs in its highest frequencies amongst livestock-keepers without agriculture or recent livestock-keepers without agriculture, irrespective of the language group.

People have retained the ability to digest milk in the western, drier half of southern Africa for at least the last 1300 years (Breton et al., 2014). This area is unsuitable for the cultivation of the indigenous summer rainfall crops, sorghum and millet, due to the low rainfall. This confirms Simoons (1970, 695) argument that the "Low incidence of intolerance, it is held, would develop over time in a group that has an abundant milk supply, that has alternate foodstuffs inadequate in amount and quality, and that consumes milk in lactose-rich forms." The contemporary distribution of groups with lactase persistence matches the distribution of Later Stone Age sites with evidence of livestock (Figure 6.5) - confirming the strong 


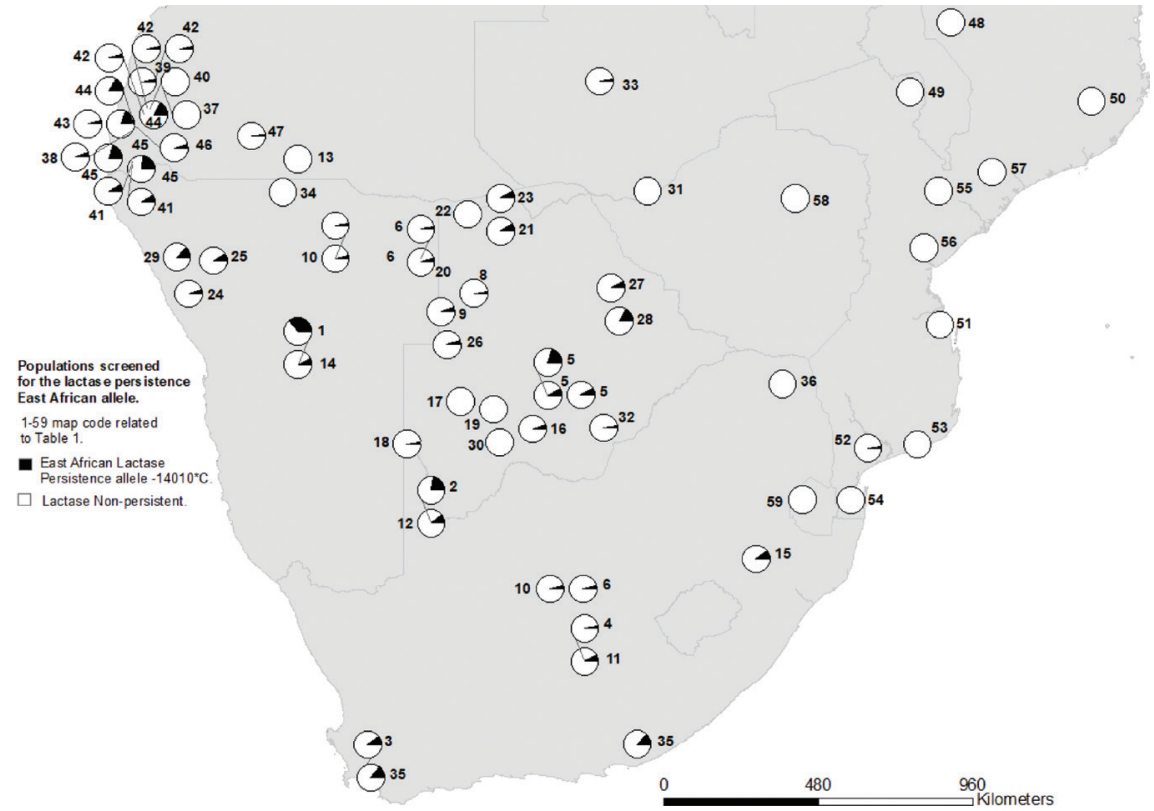

FIGURE 6.3 Map showing the distribution of the East African lactase persistence allele in Southern African

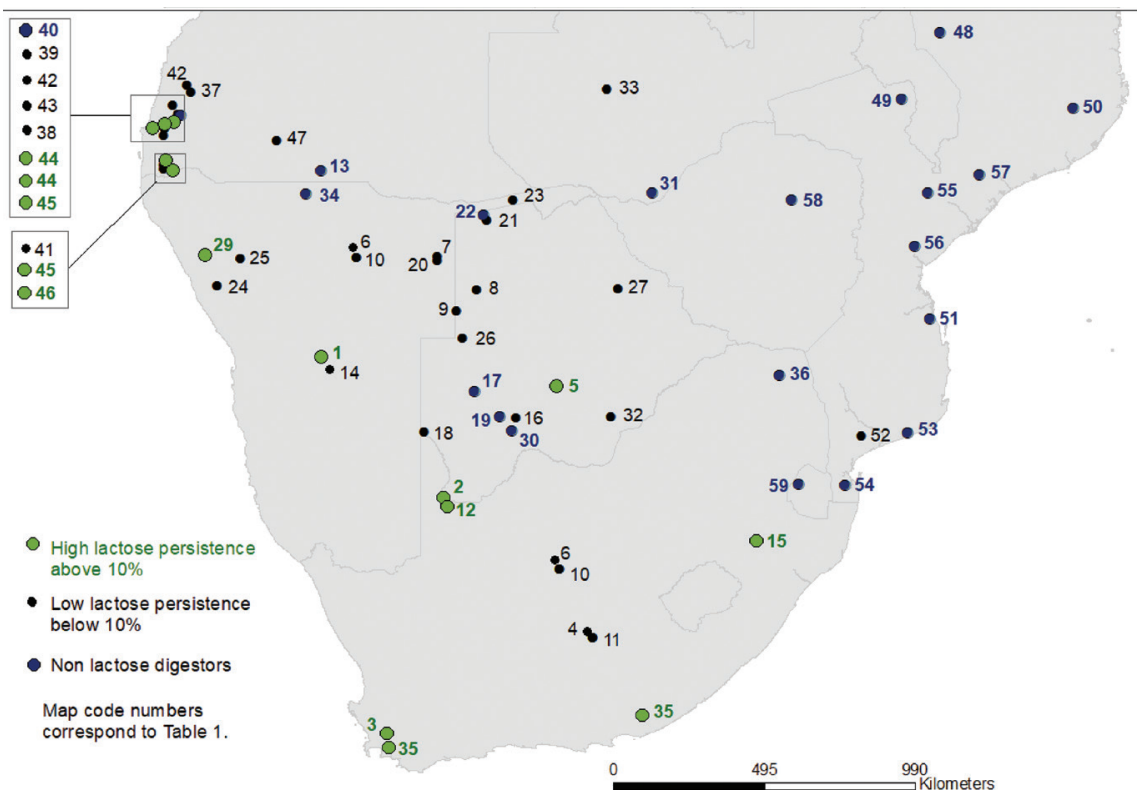

FIGURE 6.4 Map showing Southern African populations with the highest prevalence of LP Allele $-14010^{*} \mathrm{C}$ 


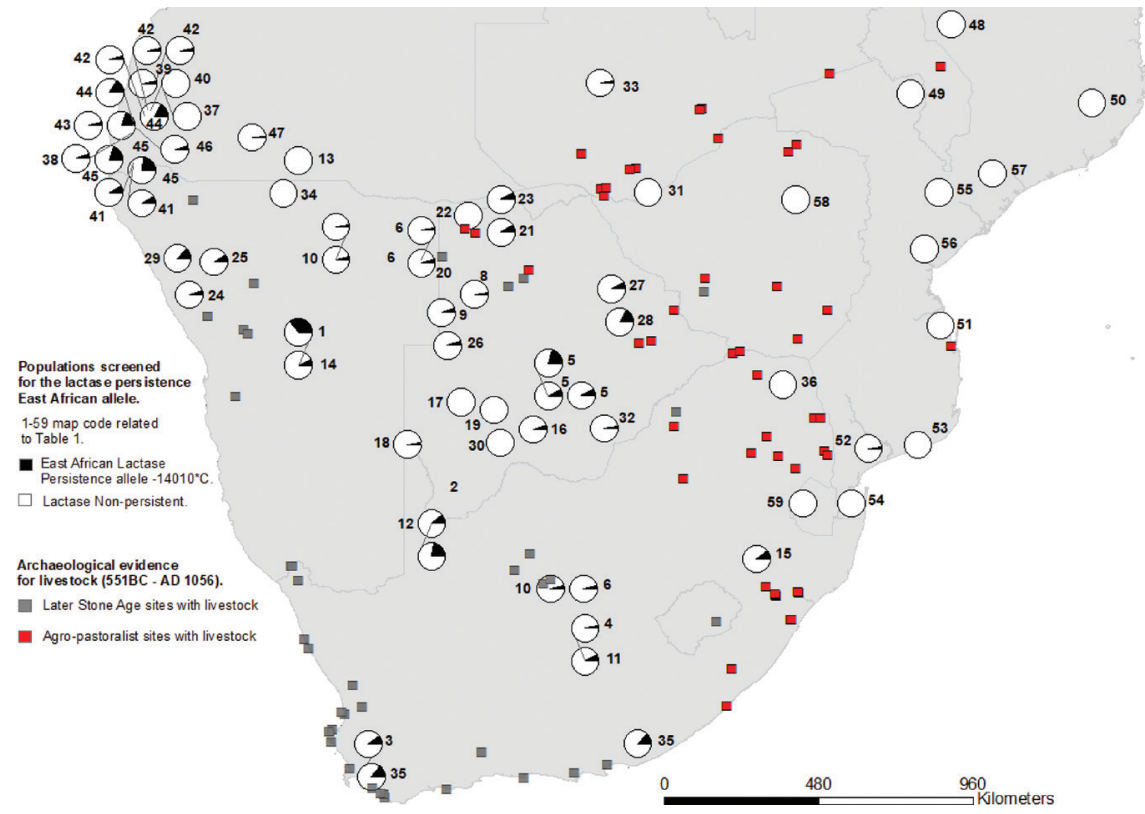

FIGURE 6.5 Archaeological evidence for livestock-keeping with and without agriculture compared to the distribution of the lactase persistence allele

Present-day distribution of African Trypanosomiasis.

Distribution of African Trypanosomiasis (after Gifford-Gonzalez 1990)

Line dividing areas where Trypanosoma brucei

gambiense and Tryanosoma brucei rhodesiense occur (after Brun et al. 2011).

Archaeological evidence for livestock (551BC-AD 1056).

- LSA Inestock sites.

agro-pastoralist livestock sites.

Present-day southern African populations screened for lactase persistence.

(3) Frequency of the $-14010^{*} \mathrm{C}$ East African LP allele.

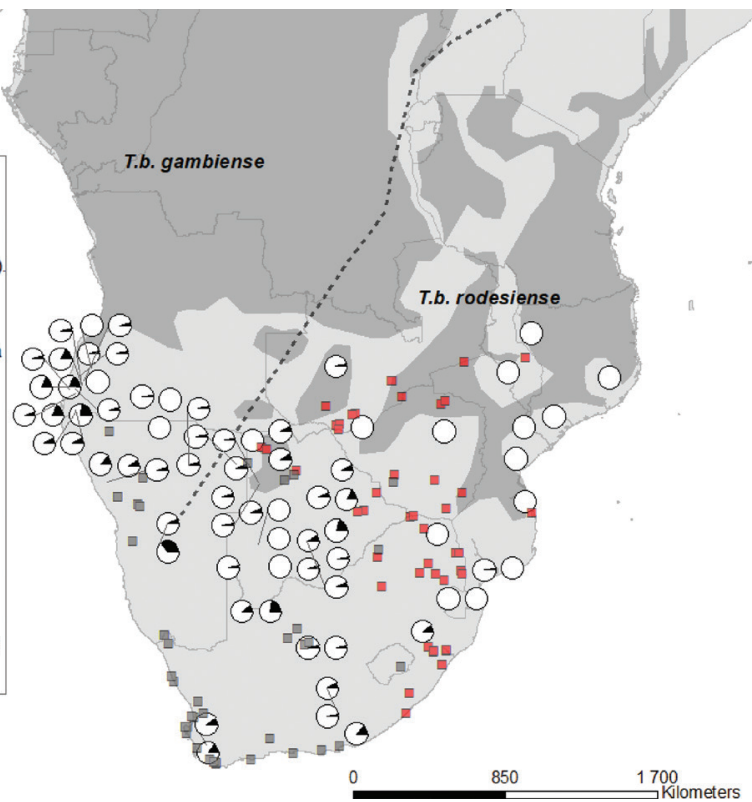

FIGURE 6.6 Archaeological evidence for livestock-keeping at approximately $1000-1200 \mathrm{BP}$ and the lactase persistence allele overlaid on the present-day distribution of African trypanosomiasis 
association of the ability to digest lactose with pastoralism (Holden and Mace, 1997). The ethnographic and historic record of milk consumption - in its fresh lactose-rich form - amongst the Khoe supports this association (Lombard and Parson, 2015).

Unique to the southernmost part of southern Africa is a winter rainfall zone that stretches from the Cape northwards into Namibia (Figure 6.1). Seasonal movement across its boundaries to the summer rainfall zone would have provided pastoralists with all year round rainfall that would be necessary for specialized milch pastoralism to flourish, mimicking the bimodal rainfall that is seen as central to the rise of pure milch pastoralism in East Africa 3000 years ago (Marshall, 199o; Marchant et al., 2018; Russell, 2020).

2. Southern Africa's Bantu-language speaking groups have a low incidence of the allele as compared to Khoe-speaking groups.

This low incidence might reflect (1) the absence of a history of livestock-keeping. The trypanosomiasis belt excludes much of tropical Africa as a cattle-keeping area (Simoons, 1974). Where it is endemic in southern Africa we find Bantu-language speaking matrilineal farmers without livestock (Holden and Mace, 2003) (Figure 6.6), or (2) the absence of fresh milk consumption rather than the absence of livestock. The cultural practice of drinking sour milk products is well documented amongst southern African Bantu-speaking peoples (Table 6.2). It is hard to find any record of the consumption of fresh milk by southern Bantu-language speakers with the exception of herd boys, who may drink milk directly from cows when out herding, and fresh milk sometimes given to children. Fermented, sour milk products have a reduced lactose content making them more digestible to lactase deficient groups (Holden and Mace, 1997). Segal et al. (1983), in their study of lactase persistence in southern African population groups show that raw milk, fermented in a gourd in the traditional way, contains $2.6 \%$ lactose, compared to the $4.7 \%$ lactose of full cream fresh milk. Thirdly, (3) those southern African Bantu-language speakers without the LP allele might represent a demic migration from the area of northern Angola, Gabon, and Congo in a southeastward direction towards South Africa, rather than from an origin in East Africa. The archaeological evidence, whilst fairly robust for the connection between East Africa and South Africa (Parkington and Hall, 2012), is unhelpful for tracing connections to western-central Africa. Such evidence for a western stream of demic migration from Angola to South Africa, as suggested by Huffman (2007) on the basis of pottery styles is weak and must be revisited (Parkington and Hall, 2012; Lander 
TABLE 6.2 List of ethnographic and historic accounts of the consumption of milk amongst Southern African groups

\begin{tabular}{|c|c|c|c|c|}
\hline Group & Country & $\begin{array}{l}\text { Sour cows's } \\
\text { milk }\end{array}$ & $\begin{array}{l}\text { Fresh cows's } \\
\text { milk }\end{array}$ & Reference \\
\hline Swazi & Eswatini & $\begin{array}{l}\text { Emasi (mainly } \\
\text { drunk by } \\
\text { children) }\end{array}$ & $\begin{array}{l}\text { Herdboys } \\
\text { in the veld } \\
\text { milk directly } \\
\text { into their } \\
\text { mouths } \\
\text { (Jones 1963: } \\
75 \text { ) }\end{array}$ & $\begin{array}{l}\text { Simatende et al. } \\
2015 ; \\
\text { Kuper 1986: 44; } \\
\text { Jones } 1963\end{array}$ \\
\hline Xhosa & $\begin{array}{l}\text { Eastern Cape, South } \\
\text { Africa }\end{array}$ & Amasi & $\begin{array}{l}\text { Milk 'always } \\
\text { used sour' } \\
\text { (Hunter } \\
\text { 1961:105) }\end{array}$ & $\begin{array}{l}\text { Beukes et al. 2001; } \\
\text { Hunter 1961; } \\
\text { Shaw and van } \\
\text { Wermelo 1974: } \\
247,250\end{array}$ \\
\hline Zulu & South Africa & Amasi & $\begin{array}{l}\text { Hardly } \\
\text { ever drank } \\
\text { "green milk" }\end{array}$ & \\
\hline \multirow[t]{3}{*}{$\begin{array}{l}\text { Southern } \\
\text { Sotho }\end{array}$} & South Africa & Mafi & & Beukes et al. 2001 \\
\hline & Botswana & Madila & & $\begin{array}{l}\text { Ohiokpehai and } \\
\text { Jagow } 1998\end{array}$ \\
\hline & $\begin{array}{l}\text { South Africa } \\
\text { Zambia }\end{array}$ & $\begin{array}{l}\text { Sethemi } \\
\text { Mabisi }\end{array}$ & & $\begin{array}{l}\text { Kebede } \text { et al. } 2007 \\
\text { Jans et al. } 2017\end{array}$ \\
\hline Nharo & Ghanzi, Botswana & & $\begin{array}{l}\text { Fresh goats' } \\
\text { milk }\end{array}$ & Guenther 1986 \\
\hline Gwi & Kutse, Botswana & & $\begin{array}{l}\text { Fresh goats' } \\
\text { milk }\end{array}$ & $\begin{array}{l}\text { Ikeya 1993, } \\
\text { Sugwara } 1991\end{array}$ \\
\hline Gana & Kutse, Botswana & & $\begin{array}{l}\text { Fresh goats' } \\
\text { milk }\end{array}$ & $\begin{array}{l}\text { Ikeya 1993, } \\
\text { Sugwara 1991 }\end{array}$ \\
\hline $\begin{array}{l}\text { Hunter- } \\
\text { gatherer } \\
\text { stock- } \\
\text { keepers }\end{array}$ & Nyae Nyae, Nambia & $\begin{array}{l}\text { Sour milk } \\
\text { products }\end{array}$ & & $\begin{array}{l}\text { Marshall and } \\
\text { Ritchie, } 1984\end{array}$ \\
\hline Nama & $\begin{array}{l}\text { Richtersveld, Northern } \\
\text { Cape, South Africa }\end{array}$ & Soured milk & & Schapera 1930 \\
\hline
\end{tabular}


and Russell, 2018). The Kalundu pottery tradition of the western stream, purporting to link pottery found at the coastal midden site of Benfica, in Angola to sites in the eastern half of South Africa, includes very few sites and the basis of the argument is unclear (cf. Huffman, 2007) (Table 6.3).

And lastly, (4) it might reflect interaction and proximity with a milch pastoralist group. The high incidence of the LP allele among the Xhosa, Sotho-Tswana and Zulu agro-pastoralists is suggestive of a long history of interaction between their ancestral groups and Khoe pastoralists. These Bantu-language speaking agro-pastoralists lived close to the historically known territory of Khoe pastoralists along the natural boundary to farmer expansion, the summer rainfall boundary. This boundary is also seen in the archaeological distribution of LSA sites as compared to farmer sites (Figure 6.1) (Parking and Hall, 2012). Genetic and linguistic studies reflect a similar pattern of the long interaction of Khoisan and Bantu-speaking groups (Pakendorf et al., 2017).

3. Non-Khoe-speaking hunter-gatherers have low levels of the allele. This is not unexpected as this is one of the few examples of foragers who resisted and rejected the more labour intensive economies of animal domestication and crop production (Russell and Lander, 2015). More interesting and requiring further investigation is why Khoe-speaking hunter-gatherers, in particular, have the LP allele. Examples of hunter-gatherers with the LP allele, in frequencies of up to $20 \%$, are the Khoe-speaking Gui and Gana of the Kutse Game Reserve, in central Botswana (Table 6.1). Although they live by hunting and gathering, their livestock keeping is well-documented (Ikeya, 1993; Osaki, 1984; 199o; Sugawara, 1991; Tanaka, 1969, 1976). Livestock, predominately goats, are never slaughtered but are kept as a social rather than a subsistence strategy, to build alliances and to use in economic exchanges (Russell, 2017). The low levels of livestock at LSA sites might reflect such a livestock-keeping and circulating system. Milking would fit easily within such a system, although little milking was recorded amongst these ethnographically observed hunter-gatherer groups (Ikeya (1993) records $200 \mathrm{ml}$ of milk being collected on a particular day), there is evidence of them following milking practices. For example, young animals are separated from their mothers during the day and dung is applied to their udders to deter feeding. Goat-keeping within a similar system is also mentioned by Guenther (1986), who notes the drinking of fresh goats' milk among the Nharo of Ghanzi, Botswana. 
TABLE 6.3 Sites with pottery which fit Huffman's (2007) western stream of demic diffusion among Bantu-language speaking farmers

\begin{tabular}{|c|c|c|c|c|}
\hline $\begin{array}{l}\text { Site, Country, site } \\
\text { type }\end{array}$ & $\begin{array}{l}\text { Radiocarbon } \\
\text { date }\end{array}$ & $\begin{array}{l}\text { Calibrated } \\
\text { Date }\end{array}$ & $\begin{array}{l}\text { Western stream, } \\
\text { kalundu tradition, } \\
\text { pottery type }\end{array}$ & Reference \\
\hline $\begin{array}{l}\text { Benfica, Angola } \\
\text { Coastal shell } \\
\text { midden }\end{array}$ & $\begin{array}{l}1810 \pm 50 \\
\text { Pta-212 }\end{array}$ & AD $212-322$ & Kalundu & $\begin{array}{l}\text { Dos Santos } \\
\text { and Ervedosa } \\
\text { 1970; Vogel } \\
\text { and Marais } \\
\text { 1971; Huffman } \\
\text { 2005, } 2007\end{array}$ \\
\hline $\begin{array}{l}\text { Gundu, Zambia } \\
\text { Inland open-air }\end{array}$ & $\begin{array}{l}1510 \pm 85 \\
G X-1114\end{array}$ & $\mathrm{AD} 48 \mathrm{o}-658$ & $\begin{array}{l}\text { Kamangoza type } \\
\text { pottery showing } \\
\text { affiliation } \\
\text { to Kalundu, } \\
\text { Dambwa and } \\
\text { Kumadzulo ware } \\
\text { and has origins } \\
\text { with Naviundu } \\
\text { pottery in the } \\
\text { Congo. }\end{array}$ & $\begin{array}{l}\text { Huffman 1989, } \\
2007\end{array}$ \\
\hline
\end{tabular}

\begin{tabular}{|c|c|c|c|c|}
\hline $\begin{array}{l}\text { Wosi, South } \\
\text { Africa } \\
\text { Inland open-air } \\
\text { riverside }\end{array}$ & $\begin{array}{l}1460 \pm 50 \\
\text { Pta-410o }\end{array}$ & AD 592-662 & Msuluzi & $\begin{array}{l}\text { Van } \\
\text { Schalkwyk } \\
1994\end{array}$ \\
\hline $\begin{array}{l}\text { Lydenburg Head } \\
\text { site, South Africa } \\
\text { Inland open-air }\end{array}$ & $\begin{array}{l}1460 \pm 50 \\
\text { Pta-328 }\end{array}$ & AD $592-662$ & $\begin{array}{l}\text { Kalundu or } \\
\text { Matola }\end{array}$ & $\begin{array}{l}\text { Maggs } \\
\text { 1980, Evers } \\
\text { et al. 1982, } \\
\text { Whitelaw } 1996\end{array}$ \\
\hline $\begin{array}{l}\text { Zambezi Farm, } \\
\text { Zambia } \\
\text { Inland open-air }\end{array}$ & $\begin{array}{l}1410 \pm 130 \\
N-1140\end{array}$ & AD $544-856$ & $\begin{array}{l}\text { Pottery similar } \\
\text { to Kalundu, } \\
\text { Dambwa and } \\
\text { Kumadzulo ware. }\end{array}$ & Vogel 1973 \\
\hline $\begin{array}{l}\text { Mhlopeni, South } \\
\text { Africa } \\
\text { Inland open-air } \\
\text { riverside }\end{array}$ & $\begin{array}{l}1400 \pm 50 \\
\text { Pta-2878 }\end{array}$ & AD $636-765$ & Msuluzi & $\begin{array}{l}\text { Maggs and } \\
\text { Ward } 1984\end{array}$ \\
\hline
\end{tabular}


TABLE 6.3 Sites with pottery which fit Huffman's (2007) western stream of demic diffusion among Bantu-language speaking farmers (cont.)

$\begin{array}{llll}\text { Site, Country, site } & \text { Radiocarbon } & \text { Calibrated } & \text { Western stream, Reference } \\ \text { type } & \text { date } & \text { Date } & \begin{array}{l}\text { kalundu tradition, } \\ \text { pottery type }\end{array}\end{array}$

\begin{tabular}{|c|c|c|c|c|}
\hline $\begin{array}{l}\text { Divuyu, } \\
\text { Botswana } \\
\text { Inland open-air }\end{array}$ & $\begin{array}{l}1400 \pm 70 \\
\text { Beta-1326o }\end{array}$ & AD $635^{-766}$ & Divuyu & $\begin{array}{l}\text { Turner 1987; } \\
\text { Denbow } 2011\end{array}$ \\
\hline $\begin{array}{l}\text { KwaGandaganda, } \\
\text { South Africa } \\
\text { Inland open-air } \\
\text { riverside }\end{array}$ & $\begin{array}{l}1395 \pm 6 \text { o } \\
\text { Wits-1918 }\end{array}$ & AD $639-765$ & Msuluzi & $\begin{array}{l}\text { Whitelaw } 1994 \\
\text { a,b. }\end{array}$ \\
\hline
\end{tabular}

$\begin{array}{lcccl}\begin{array}{l}\text { Mamba, South } \\ \text { Africa }\end{array} & \begin{array}{l}1390 \pm 50 \\ \text { Pta-4093 }\end{array} & \text { AD 643-765 } & \text { Msuluzi } & \begin{array}{l}\text { Van } \\ \text { Schalkwyk }\end{array} \\ \begin{array}{l}\text { Inland open-air } \\ \text { riverside }\end{array} & & & 1994 \\ \text { Msuluzi } & 1370 \pm 30 & \text { AD 654-763 } & \text { Msuluzi } & \text { Maggs 1980 } \\ \begin{array}{l}\text { Confluence, } \\ \text { South Africa }\end{array} & \text { Pta-2193 } & & & \\ \begin{array}{l}\text { Inland open-air } \\ \text { riverside }\end{array} & & & \\ \end{array}$

\begin{tabular}{|c|c|c|c|c|}
\hline $\begin{array}{l}\text { Magogo, South } \\
\text { Africa } \\
\text { Inland open-air } \\
\text { riverside }\end{array}$ & $\begin{array}{l}136 \mathrm{o} \pm 50 \\
\text { Pta-2874 }\end{array}$ & AD $659-765$ & Msuluzi & Maggs 1984 \\
\hline $\begin{array}{l}\text { Magarape, } \\
\text { Botswana } \\
\text { Inland open-air } \\
\text { riverside }\end{array}$ & $\begin{array}{l}135 \mathrm{O} \pm 8 \mathrm{O} \\
\mathrm{KN}-2641\end{array}$ & AD $648-841$ & $\begin{array}{l}\text { Mzonjani type } \\
\text { pottery or } \\
\text { Kalundu type } \\
\text { pottery. }\end{array}$ & $\begin{array}{l}\text { Cambpell } \\
\text { et al. 1996; } \\
\text { Huffman } 2009\end{array}$ \\
\hline $\begin{array}{l}\text { Mpame, South } \\
\text { Africa } \\
\text { Coastal open-air }\end{array}$ & $\begin{array}{l}1340 \pm 6 o \\
\text { Pta-2019 }\end{array}$ & AD $657-830$ & Msuluzi & $\begin{array}{l}\text { Vogel and Fuls } \\
1999\end{array}$ \\
\hline $\begin{array}{l}\text { Bisoli, Botswana } \\
\text { Inland open-air }\end{array}$ & $\begin{array}{l}1340 \pm 60 \\
\text { Wits-1099 }\end{array}$ & AD $657-830$ & Bisoli & $\begin{array}{l}\text { Denbow and } \\
\text { Wilmsen 1986; } \\
\text { Campbell } \\
\text { et al. 1996; } \\
\text { Huffman } 2007\end{array}$ \\
\hline
\end{tabular}


TABLE 6.3 Sites with pottery which fit Huffman's (2007) western stream of demic diffusion among Bantu-language speaking farmers (cont.)

$\begin{array}{llll}\begin{array}{l}\text { Site, Country, site } \\ \text { type }\end{array} & \begin{array}{l}\text { Radiocarbon } \\ \text { date }\end{array} & \begin{array}{l}\text { Calibrated } \\ \text { Date }\end{array} & \begin{array}{l}\text { Western stream, } \\ \text { kalundu tradition, } \\ \text { pottery type }\end{array}\end{array}$

\begin{tabular}{lllll}
\hline $\begin{array}{l}\text { Nqoma, } \\
\text { Botswana }\end{array}$ & $\begin{array}{l}1290 \pm 60 \\
\text { Beta-13257 }\end{array}$ & AD 685-860 & Divuyu and Xaro & $\begin{array}{l}\text { Wilmsen 1989, } \\
\text { 2011; Denbow }\end{array}$ \\
$\begin{array}{l}\text { Inland open-air } \\
\text { Ntsitsana, South }\end{array}$ & $\begin{array}{lll}1290 \pm 50 \\
\text { Africa }\end{array}$ & AD 685-858 & Mzuluzi and & Prins and \\
$\begin{array}{l}\text { Inland open-air } \\
\text { riverside }\end{array}$ & & Ndondondwane & Granger 1993 \\
\hline
\end{tabular}

$\begin{array}{lllll}\begin{array}{l}\text { Nanda, South } \\ \text { Africa }\end{array} & \begin{array}{l}1275^{ \pm 60} \\ \text { Wits-1917 }\end{array} & \text { AD 690-880 } & \text { Msuluzi } & \text { Whitelaw } 1993 \\ \begin{array}{l}\text { Inland open-air } \\ \text { riverside }\end{array} & & & \\ \begin{array}{l}\text { Kulubele, South } \\ \text { Africa }\end{array} & 1250 \pm 40 & \text { AD } 773^{-881} & \begin{array}{l}\text { Mzuluzi } \\ \text { pottery and/or }\end{array} & \begin{array}{l}\text { Binneman } \text { et } \\ \text { Pta-5865 } 1992\end{array} \\ \begin{array}{l}\text { Inland open-air } \\ \text { riverside }\end{array} & & \text { Ndondondwane } & \\ \end{array}$

\begin{tabular}{|c|c|c|c|c|}
\hline $\begin{array}{l}\text { Kanono Mulapo, } \\
\text { Namibia } \\
\text { shell midden site }\end{array}$ & $\begin{array}{l}1230 \pm 50 \\
\text { Pta- } 8656\end{array}$ & AD 770-96o & $\begin{array}{l}\text { Kalomo pottery } \\
\text { showing } \\
\text { similarities to } \\
\text { Kalundu and } \\
\text { Gundu }\end{array}$ & $\begin{array}{l}\text { Kinahan 2013; } \\
\text { Huffman } 1989\end{array}$ \\
\hline $\begin{array}{l}\text { Ndondondwane, } \\
\text { South Africa } \\
\text { Inland open-air } \\
\text { riverside }\end{array}$ & $\begin{array}{l}122 \mathrm{O} \pm 50 \\
\text { Pta-238 }\end{array}$ & AD $774-819$ & Ndondondwane & $\begin{array}{l}\text { Maggs } \\
\text { 1984, Van } \\
\text { Schalkwyk et } \\
\text { al. } 1997\end{array}$ \\
\hline $\begin{array}{l}\text { SK17, South } \\
\text { Africa } \\
\text { Inland open-air }\end{array}$ & $\begin{array}{l}1210 \pm 50 \\
\text { Pta-35O7 }\end{array}$ & AD $777-967$ & $\begin{array}{l}\text { Garonga pottery } \\
\text { or Kalundu } \\
\text { (Ndondondwane/ } \\
\text { Lydenburg) } \\
\text { pottery }\end{array}$ & $\begin{array}{l}\text { Meyer 1984; } \\
\text { Plug 1989; } \\
\text { Huffman 2007 }\end{array}$ \\
\hline $\begin{array}{l}\text { Kalundu Mound, } \\
\text { Zambia } \\
\text { Inland open-air }\end{array}$ & $\begin{array}{l}1160 \pm 90 \\
\text { SR-41 }\end{array}$ & AD $780-1020$ & Kalundu & Fagan 1967 \\
\hline
\end{tabular}


TABLE 6.3 Sites with pottery which fit Huffman's (2007) western stream of demic diffusion among Bantu-language speaking farmers (cont.)

$\begin{array}{llll}\text { Site, Country, site } & \text { Radiocarbon } & \text { Calibrated } & \text { Western stream, Reference } \\ \text { type } & \text { date } & \text { Date } & \begin{array}{l}\text { kalundu tradition, } \\ \text { pottery type }\end{array}\end{array}$

\begin{tabular}{|c|c|c|c|c|}
\hline $\begin{array}{l}\text { Dombashaba, } \\
\text { Botswana } \\
\text { Inland hilltop }\end{array}$ & $\begin{array}{l}1150 \pm 80 \\
\mathrm{I}-13746\end{array}$ & AD 859-1024 & Bisoli & $\begin{array}{l}\text { Huffman } \\
2005,2007\end{array}$ \\
\hline Ntshekane, South & $1150 \pm 45$ & AD $893-989$ & Ntshekane & Maggs and \\
\hline $\begin{array}{l}\text { Africa } \\
\text { Inland open-air } \\
\text { riverside }\end{array}$ & Pta-1058 & & & Michael 1976 \\
\hline
\end{tabular}

\begin{tabular}{|c|c|c|c|c|}
\hline $\begin{array}{l}\text { Kamangoza, } \\
\text { Zambia } \\
\text { Inland open-air }\end{array}$ & $\begin{array}{l}1015^{ \pm 105} \\
N-419\end{array}$ & AD $987-1185$ & $\begin{array}{l}\text { Kamangoza } \\
\text { pottery showing } \\
\text { affiliation } \\
\text { to Kalundu, } \\
\text { Dambwa and } \\
\text { Kumadzulo ware. }\end{array}$ & Vogel 1971 \\
\hline $\begin{array}{l}\text { Isamu Pati, } \\
\text { Zambia } \\
\text { Inland open-air }\end{array}$ & $\begin{array}{l}910 \pm 90 \\
\text { SR-31 }\end{array}$ & $\begin{array}{l}\text { AD 1046- } \\
1265\end{array}$ & $\begin{array}{l}\text { Kalomo pottery } \\
\text { showing } \\
\text { similarities to } \\
\text { Kalundu and } \\
\text { Gundu (Naviundu } \\
\text { from Congo } \\
\text { (western origin) } \\
\text { is an ancestor to } \\
\text { Gundu pottery) }\end{array}$ & $\begin{array}{l}\text { Huffman 1989, } \\
2005\end{array}$ \\
\hline
\end{tabular}

\section{Conclusions}

The archaeological distribution for livestock remains over the last 2100 years shows the predominance of cattle-keeping among farmers in the eastern half of the sub-continent and sparser, yet continuous, caprine-keeping among Later Stone Age livestock-keepers in the low rainfall areas to the west. We might at a first glance expect that lactase persistence might dominate on the 
eastern side of southern Africa. Genetic research shows that the reverse is true. The east African lactase persistence allele, $-14010^{*} \mathrm{C}$, is overwhelmingly found among pastoralist people in the western parts, irrespective of their language group. To explain this pattern, we turn to the ethnographic and historic record, which show the cultural practice among Bantu-speakers of drinking milk only in its fermented, lactose- reduced form. This is sometimes through its spontaneous fermentation in a gourd, at room temperature over a number of days, or through the addition of certain plants. Ethnography helps to explain why some hunter-gatherers have a high incidence of lactase persistence: they are those who have seen the social value of livestock in exchange networks, with small quantities of milk drinking. They remain overwhelmingly foragers for subsistence.

Archaeological evidence for the large herds of Khoe-owned cattle, observed historically, from the late 16th century onwards in the western and eastern Cape, which drew sailors and then settlers to southern Africa, has not been found. The archaeological picture is incomplete. For example, from $165^{2}$ to 1699, careful mercantile records show that 20 ooo cattle and 40 ooo sheep were traded with the Cape Khoe by the passing ships of the Dutch East India Company (voc) (Ross, 2012). The contrast with the total 21 cattle and 365 caprine bones found across the entire 2100 year period of the archaeology of the Later Stone Age livestock-keepers, is useful as an example of just how fragmentary the archaeological record can be (Russell and Lander 2015).

There are unresolved differences between and within the genetic and the archaeological findings. For example, the timing of the arrival of the LP allele, $-14010^{*} \mathrm{C}$, is estimated to be at 1300 years BP by geneticists (Breton et al., 2014). This is 800 years later than the earliest archaeological discovery of remains of livestock in this region. On the basis of Y chromosomal evidence, Henn et al. (2009) estimate that pastoralism arrived in southern Africa from eastern Africa at around 2000 years ago. How accurate are the genetic clock estimations? Why do they differ? The ethnic identities of modern day southern African populations are complicated and complex: it would be useful to attempt to re-trace the histories of those groups sampled by geneticists.

Yet it is only by boldly confronting and challenging discrepancies between and within different disciplines that a fuller understanding of the complex history of Africa's past will be achieved. And what satisfaction when, as in the recognition of the importance of milch pastoralism in the drier western half of southern Africa for over a millennium by scholars from genetics, ethnography and archaeology, they concur. 


\section{References}

Alves, I., Coelho, M., Gignoux, C., Damasceno, A., Prista, A., and Rocha, J. 2011. Genetic homogeneity across Bantu-speaking groups from Mozambique and Angola challenges early split scenarios between East and West Bantu populations. Human Biology 83(1): 13-38. https://doi.org/10.3378/027.083.0102.

Beukes, E.M., Bester, B.H., and Mostert, J.F. 2001. The microbiology of South African traditional fermented milks. International Journal of Food Microbiology 63(3): 189-197. https://doi.org/10.1016/So168-16o5(oo)oo417-7.

Binneman, J.N.F., Webley, L., and Biggs, V. 1992. Notes and reports: preliminary notes on an Early Iron Age site in the Great Kei River valley, Eastern Cape. Southern African Field Archaeology 1(2): 108-109.

Breton, G., Schlebusch, C.M., Lombard, M., Sjödin, P., Soodyall, H., and Jakobsson, M. 2014. Lactase persistence alleles reveal partial East African ancestry of southern African Khoe pastoralists. Current Biology 24(8): 852-858. https://doi.org/10.1016/j. cub.2014.02.041,

Brun, R., Don, R., Jacobs, R.T., Wang, M.Z., and Barrett, M.P. 2011. Development of novel drugs for human African trypanosomiasis. Future Microbiology 6(6): 677-691. https://doi.org/10.2217/fmb.11.44.

Campbell, A.C., van Waarden, C., and Holmberg, G. 1996. Variation in the Early Iron Age of southeastern Botswana. Botswana Notes and Records 1: 1-22.

Coelho, M., Sequeira, F., Luiselli, D., Beleza, S., and Rocha, J. 2009. On the edge of Bantu expansions: mtDNA, Y chromosome and lactase persistence genetic variation in southwestern Angola. BMC Evolutionary Biology 9(1): 8o. https://doi. org/10.1186/1471-2148-9-80.

Coutu, A. N., Taurozzi, A., Mackie, M., Trolle Tensen, T.Z., Collins, M. J. and Sealy, J. 2021. Palaeoproteomics confirm earliest domesticated sheep in southern Africa ca. 2000 BP. Scientific Reports 11(1): 1-11. https://doi.org/10.1038/s41598-021-85756-8.

Denbow, J. 2011. Excavations at Divuyu, Tsodilo Hills. Botswana Notes and Records 43: $76-94$.

Denbow, J.R. and Wilmsen, E.N. 1986. Advent and course of pastoralism in the Kalahari. Science $234(4783)$ : 1509-1515.

Dos Santos Júnior, J.R. and Ervedosa, C.M. 1970. A estação arqueológica de Benfica: Luanda-Angola. Ciencias Biologicas 1(1): 31-51.

Evers, T.M., Voigt, E.A., and de Villiers, H. 1982. Excavations at the Lydenburg Heads site, eastern Transvaal, South Africa. South African Archaeological Bulletin 1: 16-33.

Fagan, B.M. 1967. Iron Age cultures in Zambia (Kalomo and Kangila). Chatto and Windus: London.

Guenther, M.G. 1986. The Nharo Bushmen of Botswana: tradition and change. Helmut Buske Verlag: Hamburg, Germany. 
Henn, B.M., Gignoux, C., Lin, A.A., Oefner, P.J., Shen, P., Scozzari, R., Cruciani, F., Tishkoff, S.A., Mountain, J.L., and Underhill, P.A. 20o8. Y-chromosomal evidence of a pastoralist migration through Tanzania to southern Africa. Proceedings of the National Academy of Sciences of the USA 105(31): 10693-10698. https://doi .org/10.1073/pnas.0801184105.

Holden, C.J. and Mace, R. 1997. Phylogenetic Analysis of the Evolution of Lactose Digestion in Adults. Human Biology 81(5/6): 597-619.

Holden, C.J. and Mace, R. 2003. Spread of cattle led to the loss of matrilineal descent in Africa: a coevolutionary analysis. Proceedings of the Royal Society of London. Series B: Biological Sciences 270(1532): 2425-2433. https://doi.org/10.1098/rspb.2003.2535.

Holden, C. and Mace, R. 20og. Phylogenetic analysis of the evolution of lactose digestion in adults. Human Biology 81(5/6): 597-619.

Huffman, T.N. 1989. Iron Age migrations. Witwatersrand University Press: Johannesburg, South Africa.

Huffman, T.N. 2005. The stylistic origin of Bambata and the spread of mixed farming in southern Africa. Southern African Humanities 17(1): 57-79.

Huffman, T.N. 2007. Handbook to the Iron Age. University of KwaZulu-Natal Press: Pietermaritzburg, South Africa.

Huffman, T.N. 2009. A cultural proxy for drought: ritual burning in the Iron Age of southern Africa. Journal of Archaeological Science 36(4): 991-1005. https://doi .org/10.1016/j.jas.2008.11.026.

Hunter, M. 1961. Reaction to conquest. Effects of contact with Europeans on the Pondo of South Africa. Oxford University Press: London, UK.

Ikeya, K. 1993. Goat raising among the San in the central Kalahari. African Study Monographs 14(1): 39-52. https://doi.org/10.14989/6810o.

Jans, C., Meile, L., Kaindi, D.W.M., Kogi-Makau, W., Lamuka, P., Renault, P., Kreikemeyer, B., Lacroix, C., Hattendorf, J., Zinsstag, J., Schelling, E., Fokou, G., and Bonfoh, B. 2017. African fermented dairy products: Overview of predominant technologically important microorganisms focusing on African Streptococcus infantarius variants and potential future applications for enhanced food safety and security. International Journal of Food Microbiology 25o: 27-36. https://doi.org/10.1016/j.ijfoodmicro .2017.03.012.

Jones, S.M. 1963. A study of Swazi nutrition: report of the Swaziland Nutrition Survey 1961-62. Institute for Social Research, University of Natal: Durban, South Africa.

Jones, B.L., Oljira, T., Liebert, A., Zmarz, P., Montalva, N., Tarekeyn, A., Ekong, R., Thomas, M.G., Bekele, E., Bradman, N., and Swallow, D.M. 2015. Diversity of lactase persistence in African milk drinkers. Human Genetics 134(8): 917-925. https://doi .org/10.1007/soo439-015-1573-2.

Kebede, A, Viljoen, B.C., Gadaga, T.H., Narvhus, J.A., and Lourens-Hattingh, A. 2007. The effect of container type on the growth of yeast and lactic acid bacteria during 
production of Sethemi, South African spontaneously fermented milk. Food Research International 40: 33-38. https://doi.org/10.1016/j.foodres.2006.07.012.

Kinahan, J. 1991. Pastoral nomads of the central Namib Desert: the people that time forgot. Namibia Archaeological Trust and New Namibia Books: Windhoek, Namibia.

Kinahan, J. 1994. A new archaeological perspective on nomadic pastoralist expansion in south-western Africa. Azania: Archaeological Research in Africa 29(1): 211-226. https://doi.org/10.108o/oo672709409511677.

Kinahan, J. 2013. The Acquisition of ceramics by hunter-gatherers on the Middle Zambezi in the first and second millennium AD. Journal of African Archaeology 11(2): 197-209. https://doi.org/10.3213/2191-5784-10243.

Kuper, H. 1986. The Swazi. A South African kingdom. Holt, Rinehart and Winston: London.

Lander, F. and Russell, T. 2018. The archaeological evidence for the appearance of pastoralism and farming in southern Africa. PloS One 13(6): eo198941. https://doi. org/10.1371/journal.pone.0198941.

Lander, F. and Russell, T. 2020. A southern African archaeological database of organic containers and materials, 800 cal BC to cal AD 1500: Possible implications for the transition from foraging to livestock-keeping. Plos One 15(7): eo235226. https://doi. org/10.1371/journal.pone.0235226.

Lombard, M. and Parsons, I. 2015. Milk not meat: The role of milk amongst the Khoe peoples of southern Africa. Journal of African Archaeology 13(2): 149-166. https:// doi.org/10.3213/2191-5784-10272.

Ohiokpehai, O. and Jagow, J. 1998. Improving Madila-a traditional fermented milk from Botswana. Intermediate Technol Food Chain 23(6).

Orton, J. 2015. The introduction of pastoralism to southernmost Africa: Thoughts on new contributions to an ongoing debate. Azania: Archaeological Research in Africa 5o(2): 250-258. https://doi.org/10.108o/0o67270X.2015.1019262

Osaki, M. 1984. The social influence of change in hunting technique among the Kalahari San. African Study Monographs 5: 49-62.

Osaki, M. 1990. The influence of sedentism on sharing among the central Kalahari hunter-gatherers. African Study Monographs, Supplementary 12: 59-87.

Macholdt, E., Slatkin, M., Pakendorf, B., and Stoneking, M. 2014. New insights into the history of the C-14010 lactase persistence variant in Eastern and Southern Africa. American Journal of Physical Anthropology 156(4): 661-664. https://doi.org/10.1002/ ajpa.22675.

Maggs, T.1980. Msuluzi confluence: a seventh century Early Iron Age site on the Tugela River. Annals of the Natal Museum 24(1): 111-145.

Maggs, M.A. and Michael, M.A. 1976. Ntshekane: an Early Iron Age site in the Tugela Basin, Natal. Annals of the Natal Museum 22(3): 705-740. 
Maggs, T. and Ward, V. 1984. Early Iron Age sites in the Muden area of Natal. Annals of the Natal Museum 26(1):105-40.

Marchant, R., Richer, S., Boles, O., Capitani, C., Courtney-Mustaphi, C.J., Lane, P., Prendergast, M.E., Stump, D., De Cort, G., Kaplan, J.O., Phelps, L., Kay, A., Olago, D., Petek, N., Platts, P.J., Punwong, P., Widgren, M., Wynne-Jones, S., Ferro-Vázquez, C., ..., and Wright, D. 2018. Drivers and trajectories of land cover change in East Africa: Human and environmental interactions from 6000 years ago to present. EarthScience Reviews 178: 322-378. https://doi.org/10.1016/j.earscirev.2017.12.010

Marshall, F. 199o. Origins of specialized pastoral production in East Africa. American Anthropologist 92(40): 873-894. https://doi.org/10.1525/aa.1990.92.4.02aooo2o

Marshall, J. and Ritchie, C. 1984. Where are the JU/WASI of Nyae Nyae? Changes in a Bushman society: 1958-1981. Centre for African Studies, University of Cape Town: Cape Town, South Africa.

Meyer, A. 1984. A profile of the Iron Age in the Kruger national park. In: Hall, M., Avery, G., Avery, D.M., Wilson, M.L., and Humphreys, A.J.B. (eds) Frontiers: Southern African Archaeology Today. British Archaeological Reports International Series 207: Oxford, UK, pp. 215-227.

Pakendorf, B., Gunnink, H., Sands, B., and Bostoen, K. 2017. Prehistoric Bantu-Khoisan language contact: A cross-disciplinary approach. Language Dynamics and Change 7(1): 1-46. https://doi.org/10.1163/22105832-00701002

Parkington, J. and Hall, S. 2012. The appearance of food production in southern Africa 1,000 to 2,00o years ago. In: Hamilton, C., Mbenga, B.K., and Ross, R. (eds) The Cambridge History of South Africa, Volume 1: from early times to 1885. Cambridge University Press: Cambridge, UK, pp. 63-111.

Pinto, J.C., Oliveira, S., Teixeira, S., Martins, D., Fehn, A.M., Aço, T., Gayà-Vidal, M., and Rocha, J. 2016. Food and pathogen adaptations in the Angolan Namib desert: Tracing the spread of lactase persistence and human African trypanosomiasis resistance into southwestern Africa. American Journal of Physical Anthropology 161(3): 436-447. https://doi.org/10.1002/ajpa.23042

Plug, I. 1989. Aspects of life in the Kruger National Park during the early Iron Age. South African Archaeological Society: Goodwin Series 1: 62-68.

Prins, F.E., and Granger, J.E. 1993. Early farming communities in northern Transkei: the evidence from Ntsitsana and adjacent areas. Natal Museum Journal of Humanities 5(10): 153-174.

Ranciaro, A., Campbell, M.C., Hirbo, J.B., Ko, W.Y., Froment, A., Anagnostou, P., Kotze, M.J., Ibrahim, M., Nyambo, T., Omar, S.A., and Tishkoff, S.A. 2014. Genetic origins of lactase persistence and the spread of pastoralism in Africa. The American Journal of Human Genetics 94(4): 496-510. https://doi.org/10.1016/j.ajhg.2014.02.009

Russell, T. 2017. 'Where goats connect people': Cultural diffusion of livestock not food production amongst southern African hunter-gatherers during the Later Stone Age. Journal of Social Archaeology 17(2):115-137. https://doi.org/10.1177/1469605317701596 
Russell, T. 202O. The role of the Cape's unique climatic boundaries in sustaining specialized pastoralists in southern Africa during the last 2000 years. Azania: Archaeological Research in Africa 55(2): 242:257. https://doi.org/10.1080/oo67270X.2020.1757887

Russell, T. and Lander, F. 2015. 'What is consumed is wasted': From foraging to herding in the southern African Later Stone Age. Azania: Archaeological Research in Africa 5o(3): 267-317. https://doi.org/10.108o/oo67270X.2015.1079o82

Ross, R. 2012. Khoesan and immigrants: the emergence of colonial society in the Cape, 1500 - 180o. In: Hamilton, C., Mbenga, B.K., and Ross, R. (eds) The Cambridge History of South Africa, Volume 1: from early times to 1885. Cambridge University Press: Cambridge, UK, pp. 168-210.

Sadr, K. 1998. The first herders at the Cape of Good Hope. African Archaeological Review 15(2): 101-132. https://doi.org/10.1023/A:1022158701778.

Sadr, K. 2003. The Neolithic of southern Africa. Journal of African History 44: 195-209.

Sadr, K. 2004. Feasting on Kasteelberg? Early herders on the west coast of South Africa. Before Farming 3: 1-17.

Sadr, K. 2008a. Invisible herders? The archaeology of Khoekhoe pastoralists. Southern African Humanities 2O(1): 179-203.

Sadr, K. 2008b. An ageless view of first millennium AD southern African ceramics. Journal of African Archaeology 6(1): 103-129. https://doi.org/10.3213/1612-1651-10105

Sadr, K. and Sampson, C.G. 2006. Through thick and thin: early pottery in southern Africa. Journal of African Archaeology 4(2): 235-252. https://doi.org/10.3213/1612-1651-10074.

Schapera, I. 1963. The Khoisan peoples of South Africa: Bushmen and Hottentots. Routledge and Kegan Paul Limited: London, UK.

Segal, I., Gagjee, P.P., Essop, A.R., and Noormohamed, A.M. 1983. Lactase deficiency in the South African black population. The American Journal of Clinical Nutrition 38(6): 901-905. https://doi.org/10.1093/ajcn/38.6.901.

Shaw, E.M. and van Warmelo, N.J. 1974. The material culture of the Cape Nguni. Annals of the South African Museum.

Simatende, P., Gadaga, T.H., Nkambule, S.J., and Siwela, M. 2015. Methods of preparation of Swazi traditional fermented foods. Journal of Ethnic Foods 2(3): 119-125. https://doi.org/10.1016/j.jef.2015.08.0o8.

Simoons, F.J. 1970. Primary adult lactose intolerance and the milking habit: A problem in biologic and cultural interrelations. The American Journal of Digestive Diseases 15 :695-710. https://doi.org/10.1007/BFo2235991.

Smith, A.B. 2008. Pastoral origins at the Cape, South Africa: influences and arguments. Southern African Humanities $20(1)$ : 49-6o.

Smith, A.B. 2017. Why would southern African hunters be reluctant food producers? Hunter Gatherer Research 2(4): 415-435. https://doi.org/10.3828/hgr.2016.28.

Sugawara, K. 1991. The economics of social life among the central Kalahari San (G//ana-khwe and G/wikhwe) in the sedentary community at !Koi!kom. Senri Ethnological Studies 30: 91-116. http://doi.org/10.15021/oooo3135. 
Tanaka, J. 1969. The ecology and social structure of central Kalahari Bushmen: A preliminary report. Kyoto University African Studies 3: 1-26.

Tanaka, J. 1976. Subsistence ecology of Central Kalahari San. In: Lee, R.B. and DeVore, I. (eds) Kalahari hunter-gatherers: studies of the !Kung San and their neighbors. Harvard University Press: Cambridge, MA, USA, pp. 98-119.

Tishkoff, S.A., Reed, F.A., Ranciaro, A., Voight, B.F., Babbitt, C.C., Silverman, J.S., Powell, K., Mortensen, H.M., Hirbo, J.B., Osman, M., and Ibrahim, M. 2007. Convergent adaptation of human lactase persistence in Africa and Europe. Nature Genetics 39(1): 31-40. https://doi.org/10.1038/ng1946

Torniainen, S., Parker, M.I., Holmberg, V., Lahtela, E., Dandara, C., and Jarvela, I. 2009. Screening of variants for lactase persistence/non-persistence in populations from South Africa and Ghana. BMC Genetics 10: 31. https://doi.org/10.1186/1471-2156-10-31. Turner, G. 1987. Early Iron Age herders in northwestern Botswana: the faunal evidence. Botswana Notes and Records 19(1): 7-23.

Van Schalkwyk, L. 1994. Wosi: an early Iron Age village in the lower Thukela Basin, Natal. Southern African Humanities 6(10): 65-117.

Van Schalkwyk, L.O., Greenfield, H., and Jongsma, T. 1997. The early Iron Age site of Ndondondwane, Kwazulu-Natal, South Africa: preliminary report on the 1995 excavations. Southern African Field Archaeology 6(2): 61-77.

Vogel, J.O. 1971. Kamangoza: An introduction to the Iron Age cultures of the Victoria Falls region. Oxford University Press: Oxford, UK.

Vogel,J.O.1973.SomeEarlyIronAgesites in southernandwesternZambia.Azania:Archaeological Research in Africa 8(1): 25-54. https://doi.org/10.1080/00672707309511571

Vogel, J.C. and Morais, M. 1971. Pretoria Radiocarbon dates I. Radiocarbon 13: 378-394.

Vogel, J.C. and Fuls, A. 1999. Spatial distribution of radiocarbon dates for the Iron Age in southern Africa. South African Archaeological Bulletin 54: 97-101.

Whitelaw, G. 1993. Customs and settlement patterns in the first millennium AD: evidence from Nanda, an Early Iron Age site in the Mngeni Valley, Natal. Southern African Humanities 5(10): 47-81.

Whitelaw, G. 1994a. Towards an Early Iron Age worldview: some ideas from KwaZulu-Natal. Azania: Archaeological Research in Africa 29(1): 37-50.

Whitelaw, G. 1994b. KwaGandaganda: settlement patterns in the Natal Early Iron Age. Southern African Humanities 6(10): 1-64.

Whitelaw, G. 1996. Lydenburg revisited: another look at the Mpumalanga Early Iron Age sequence. South African Archaeological Bulletin 1: 75-83.

Wilmsen, E.N. 1989. The Antecedents of Contemporary Pastoralism in Western Ngamiland. Botswana Notes and Records 20: 29-39.

Wilmsen, E.N. 2011. Nqoma: an abridged review. Botswana Notes and Records 43: 95-114. 